\title{
The sigmoid volvulus: a surgical dilemma in adult patient.
}

\section{Abstract}

Sigmoid volvulus (SV) and its natural history have been recognized by physicians and surgeons since ancient Egypt times. The Papyrus Ebers mentioned that volvulus should reduce immediately or the sigmoid "colon rotted". Colonic volvulus accounts for 1-7\% of cases of large bowel obstruction in the United States and Western Europe. It generally common in elderly patients with multiple co morbidities especially neurologic conditions. Sigmoid volvulus, if complicated carries a high risk of mortality and morbidity, hence low threshold and early detection is essential. ${ }^{1-3}$ This report describes an unusual case of a very large gangrenous sigmoid volvulus in a 29 years old, young and healthy lady which undergoes traditional massage.

Keywords: sigmoid volvulus, massage, laparotomy, imaging, X-ray, ovarian cyst, abdominal pain, distended, hypovolemic shock, fluid-electrolyte imbalance, nasogastric aspiration, parenteral feeding, barium enemas, rigid sigmoidoscopy, flexible sigmoidoscopy
Volume 6 Issue 5 - 2018

\section{Sarmukh S,' Lenny SS, ${ }^{2}$ Ramesh T, ${ }^{2}$ Chew LG, ${ }^{2}$ Zaidi Z,}

'Department of Surgery, School of Medical Science, University Sains Malaysia, Malaysia

${ }^{2}$ Department of Surgery, Hospital Serdang, Selangor, Malaysia

Correspondence: Sarmukh S, Department of Surgery, Schoo of Medical Science, University Sains Malaysia, Malaysia,Tel 0179739329,Email sarmukh_rao@yahoo.com

Received: March 23, 20I8 | Published: September 24, 2018

\section{Abbreviation: SV, Sigmoid volvulus}

\section{Case report}

A 29-year-old lady, without significant medical history, presented to our hospital emergency department with 3 days history of constipation associated with generalized abdominal pain, nausea and vomiting. On further history taking, patient had a traditional massage done due to lower abdominal pain. On examination, the abdomen was distended with diffuse marked tenderness without rigidity or guarding; no hernias or masses were noted, bowel sound was sluggish. Digital rectal examination reveal normal finding. We proceeded with imaging. Abdomen X-ray shows large bowel loops are dilatation measuring about $6 \mathrm{~cm}$ and an ultrasound abdomen was done and there were free fluid presence in the abdomen and pelvis. Our impression on that time was perforated appendicitis. We proceeded with exploratory laparotomy and the intraoperative findings shows gangrenous sigmoid colon secondary to volvulus caused by broad ligament tear and twisted left ovarian cyst. We proceeded with a Hartmann's procedures. Post operative was uneventful and patient was discharge home at day five post operation (Figures 1-4).

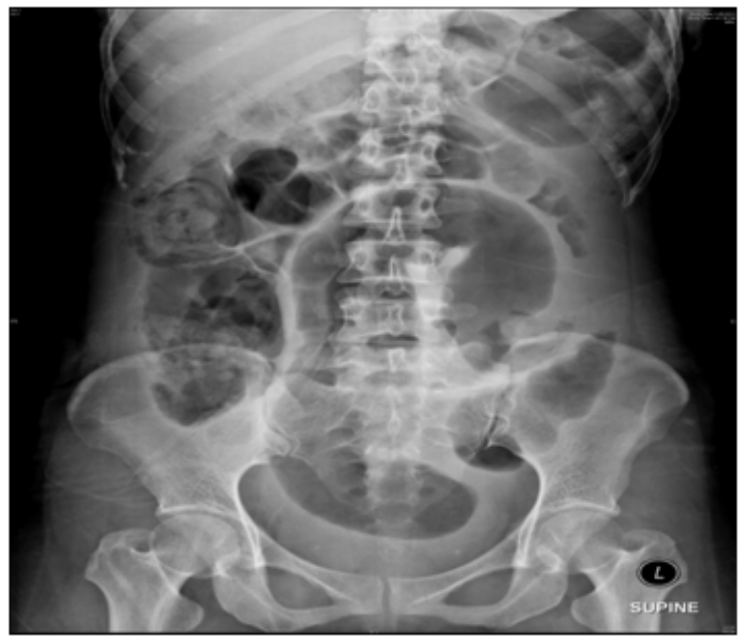

Figure I Radiological investigation abdominal x-ray shows a large bowel loops are dilatation measuring about $6 \mathrm{~cm}$.

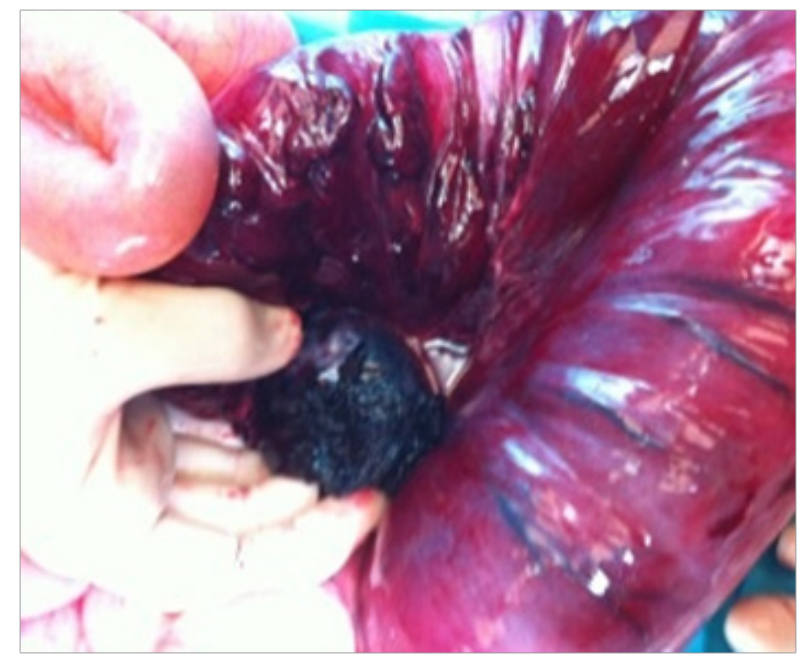

Figure 2 Intraoperative findings revealing sigmoid volvulus.

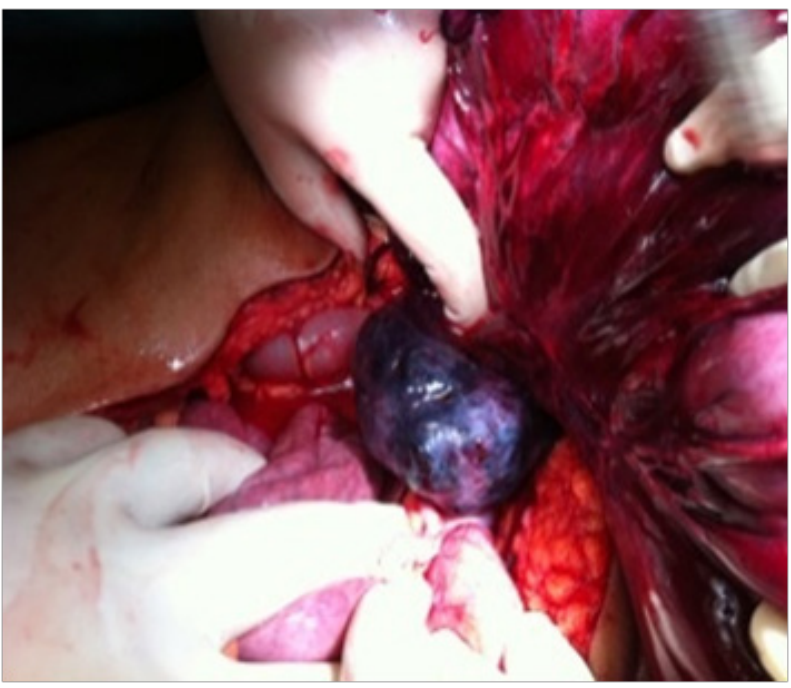

Figure 3 Intraoperative finding shows part of the redundant sigmoid colon gangrene. 


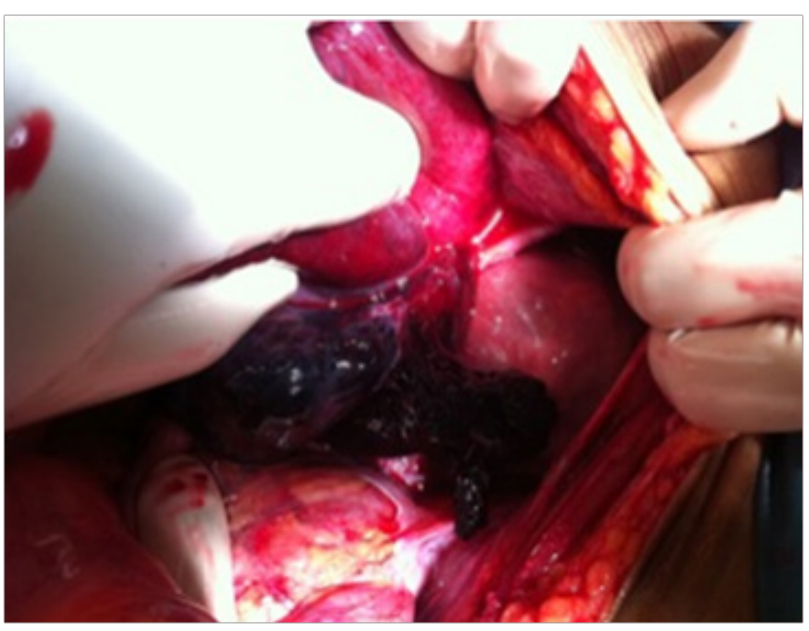

Figure 4 Intraoperative finding shows part of the redundant sigmoid colon gangrene that enters through a tear at the broad ligament and become volvulus with torsion of the left ovary.

\section{Discussion}

Colonic volvulus accounts for $1-7 \%$ of cases of large bowel obstruction in the United States and Western Europe. The colonic volvulus is more common occur in sigmoid colon which is $65-80 \%$ of cases compared with the right colon only $15-30 \%$. However, in transverse colon and splenic flexure volvulus is rare. Sigmoid volvulus, the sigmoid colon wraps around itself and its mesentery, sigmoid volvulus accounts for $2 \%$ to $50 \%$ of all colonic obstructions and has an interesting geographic dispersion. Sigmoid volvulus generally affects adults, and it is more common in males. ${ }^{1-3}$

The management approach in patient with colonic volvulus can be divided base on patient general condition whether patient is unstable, in hypovolemic shock or in septic shock. In this category of patients we need an effective resuscitation including fluid-electrolyte imbalance, nasogastric aspiration, and parenteral feeding. Nonoperative detorsion is advocated as the primary treatment choice in uncomplicated acute sigmoid volvulus. The method of non operative management is rectal tube decompression of sigmoid volvulus, barium enemas, rigid sigmoidoscopy and flexible sigmoidoscopy. Non-operative treatment is successful in $70 \%-91 \%$ of patient with complication rates of $2 \%-4.7 \%$ in elderly patients. ${ }^{4,5}$ Nevertheless, emergency surgery is indicated in minority of patients such as in peritonitis, bowel gangrene or perforation is present or in those for whom non-operative treatment is unsuccessful. Emergency surgery is associated with significant mortality and morbidity. ${ }^{6}$ The types of emergency surgery can be divided in non-definitive and definitive procedures. The definitive procedure are detorsion, sigmoidopexy, sigmoid resection with primary anastomosis and sigmoid resection with stoma creation. ${ }^{7-9}$ In our case, this patient had a gangrenous bowel and patients with volvulus proximal to the sigmoid. When the bowel is not viable, resection of bowel is mandatory. Hartmann's procedure may be lifesaving, particularly in unstable patients, and the mortality rate of the stoma procedures has been reported as low as $25 \% .^{10-11}$

\section{Conclusion}

Traditional massage can cause broad ligament tearing which lead to sigmoid volvulous. This case emphasizes the importance of early identification in the atypical patient before the appearance of twisted loop gangrene, in order to optimize patient management. Early identification and management are crucial in treating sigmoid volvulous before the appearance of gangrene and necrosis, thereby avoiding further complications and associated mortality. Emergency surgery is reserved for gangrene and failed decompression, and in patients with a high recurrence rate. Hartmann's procedures may be lifesaving, particularly in unstable patients.

\section{Acknowledgment}

None.

\section{Conflict of interest}

Author declares there is no conflict of interest.

\section{Refernces}

1. Ballantyne GH, Brandner MD, Beart RW Jr, et al. Volvulus of the colon. Incidence and mortality. Ann Surg. 1985;202(1):83-92.

2. Krupsky S, Halevy A, Orda R. Sigmoid volvulus in adolescence. J Clin Gastroenterol. 1987;9(4):467-469.

3. Hiltunen KM, Syrja H, Matikainen M. Colonic volvulus. Diagnosis and results of treatment in 82 patients. Eur J Surg. 1992;158(11-12):607-611.

4. Avots-Avotins KV, Waugh DE. Colon volvulus and the geriatric patient. Surg Clin North Am. 1982;62(2):249-260.

5. Bak MP, Boley SJ. Sigmoid volvulus in elderly patients. Am J Surg. 1986;151(1):71-75.

6. Kassi AB, Lebeau R, Yenon KS, et al. Morbidity and mortality of Hartmann's procedure for sigmoid volvulus at the University Hospital of Cocody, Abidjan. West Afr J Med. 2011;30(3):169-172.

7. Raveenthiran R, Madiba TE, Atamanalp SS, et al. Volvulus of the sigmoid colon. Colorectal Dis. 2010;12(7):e1-17.

8. Arnold GJ, Nance FC. Volvulus of the sigmoid colon. Ann Surg. 1973;177(5):527-537.

9. Atamanalp SS, Yildirgan MI, Basoglu M, et al. Sigmoid colon volvulus in children: review of 19 cases. Pediatr Surg Int. 2004;20(7):492-495.

10. Oren D, Atamanalp SS, Aydinli B, et al. An algorithm for the management of sigmoid colon volvulus and the safety of primary resection: experience with 827 cases. Dis Colon Rectum. 2007;50(4):489-497.

11. Madiba TE, Thomson SR. The management of sigmoid volvulus. JR Coll Surg Edinb. 2000;45(2):74-80. 